\title{
Programa de desarrollo de vehículos eléctricos de baterías en la UNAM
}

\author{
G. Carmona-Paredes, R. Chicurel-Uziel, E. Chicurel-Uziel y F. Gutiérrez-Martínez \\ Instituto de Ingeniería, UNAM \\ E-mail:rcu@pumas.iingen.unam.mx
}

(recibido: enero de 2002; aceptado: abril de 2002)

\begin{abstract}
Resumen
Hace ocho años, el Instituto de Ingeniería inició el proyecto de desarrollo de un minibús eléctrico de baterías, conocido ahora como el Vehículo Eléctrico UNAM. Este proyecto fue seguido de un convenio con el Gobierno del Distrito Federal para el diseño y construcción del Electrobús, un vehículo para transporte público que recientemente fue puesto en operación para ser evaluado por el Servicio de Transportes Eléctricos del DF. Dos proyectos más, enmarcados dentro del Programa de Desarrollo de Vehículos Eléctricos, se refieren a vehículos ligeros de carga como el Electrovira, caracterizado por su gran maniobrabilidad y el Vehículo Eléctrico de Reparto. Estos proyectos se realizan respectivamente en el Instituto de Ingeniería y el Centro de Diseño Mecánico de la Facultad de Ingeniería. Se ha contado también con la colaboración del Centro de Investigaciones en Diseño Industrial de la Facultad de Arquitectura, el Centro de Instrumentos y la Facultad de Química. Otros aspectos complementarios en los que se está trabajando, incluyen el desarrollo de un cargador inteligente para grandes paquetes de baterías y de un controlador electrónico dual, el estudio del desempeño dinámico de baterías de plomo-ácido, el diseño de un sistema de monitoreo de baterías y la búsqueda de nuevas alternativas de baterías.
\end{abstract}

Descriptores: vehículos eléctricos, transporte eléctrico, vehículos de reparto, baterías.

\section{Abstract}

Eight years ago, a project to develop a small battery powered electric bus, known today as "UNAM's Electric Vehicle", was started at the Engineering Institute. This project was followed by the design and construction, under an agreement with the Mexico City Government, of the "Electrobus", a public transport vehicle that was recently put in operation and is being evaluated by the City's Electric Transportation Service. Two other projects, within the scope of UNAM's Electric Vehicle Development Program refer to light trucks: the "Electrovira", characterized by exceptional maneuverability, and the "Electric Delivery Vehicle". These projects are being carried out respectively by the Engineering Institute and the School of Engineering's Center for Design and Manufacture. Other parties that have collaborated in the program are: the School of Architecture's Center for Research in Industrial Design, the Instruments Center, and the School of Chemistry. Work is also being done on complementary aspects which include the development of an intelligent charger for large battery packs and of a dual electronic controller, a study of the dynamic performance of lead-acid batteries, the design of a battery monitoring system, and the search for new battery alternatives.

Keywords: electric vehicles, electric powered transport, delivery trucks, batteries. 
DOI: http://dx.doi.org/10.22201/fi.25940732e.2002.03n3.011

\section{Introducción}

A partir de 1993, el Instituto de Ingeniería de la UNAM ha realizado proyectos relacionados con el desarrollo de vehículos eléctricos autónomos, dentro de un programa que tiene los siguientes objetivos:

1. Generar diseños propios en el área de transporte público no contaminante y crear una base tecnológica que conduzca a una etapa de fabricación masiva de vehículos de este tipo.

2. Contribuir a la formación de recursos humanos en esta área, asegurando la continuidad de las actividades de índole tecnológica y científica.

3. Fomentar la introducción de innovaciones que avancen el estado del arte a nivel mundial.

Este proyecto ha contado con valiosas aportaciones de otras dependencias universitarias, en particular, la del Centro de Diseño y Manufactura de la Facultad de Ingeniería, que se unió al programa en 1997 y con quien actualmente existe estrecha colaboración. Asimismo, participan el Centro de Investigaciones en Diseño Industrial de la Facultad de Arquitectura, el Centro de Instrumentos y la Facultad de Química.

Desde los inicios del programa se contó con el apoyo de las autoridades del Distrito Federal y también se ha recibido apoyo del Consejo Nacional de Ciencia y Tecnología para el desarrollo y pruebas de los vehículos, así como para varios aspectos del proyecto, incluyendo el desarrollo de un cargador de baterías de alta capacidad.

A continuación se describen los avances logrados en este programa.

\section{Vehículo Eléctrico UNAM}

El Vehículo Eléctrico UNAM (VE-UNAM) mostrado en la figura 1 , es un minibús con capacidad para 30 pasajeros que fue terminado en 1996. Esta unidad está equipada con un sistema hidroneumático para recuperación de energía de frenado, lo cual hace más eficiente su operación y extiende la vida de las baterías. Cuando se desea frenar, una bomba acoplada a la transmisión envía aceite a un acumulador hidroneumático, accionando un émbolo que comprime un volumen de gas. Después, al acelerar, el gas comprimido se expande y se invierte el flujo de aceite haciendo que la bomba actúe ahora como motor hidráulico, asistiendo así al motor eléctrico de tracción. Chicurel R. y Lara (1966). describen una primera versión del sistema. Chicurel R. (1999), Chicurel R. y Carmona (2001), tratan diversos aspectos de la versión que finalmente se instaló en el VE-UNAM. Aunque tales sistemas se han utilizado en autobuses con motor de combustión interna, como los descritos por Hammerström (1984) y Martini (1984), no se encontró ningún antecedente de su aplicación en vehículos eléctricos.

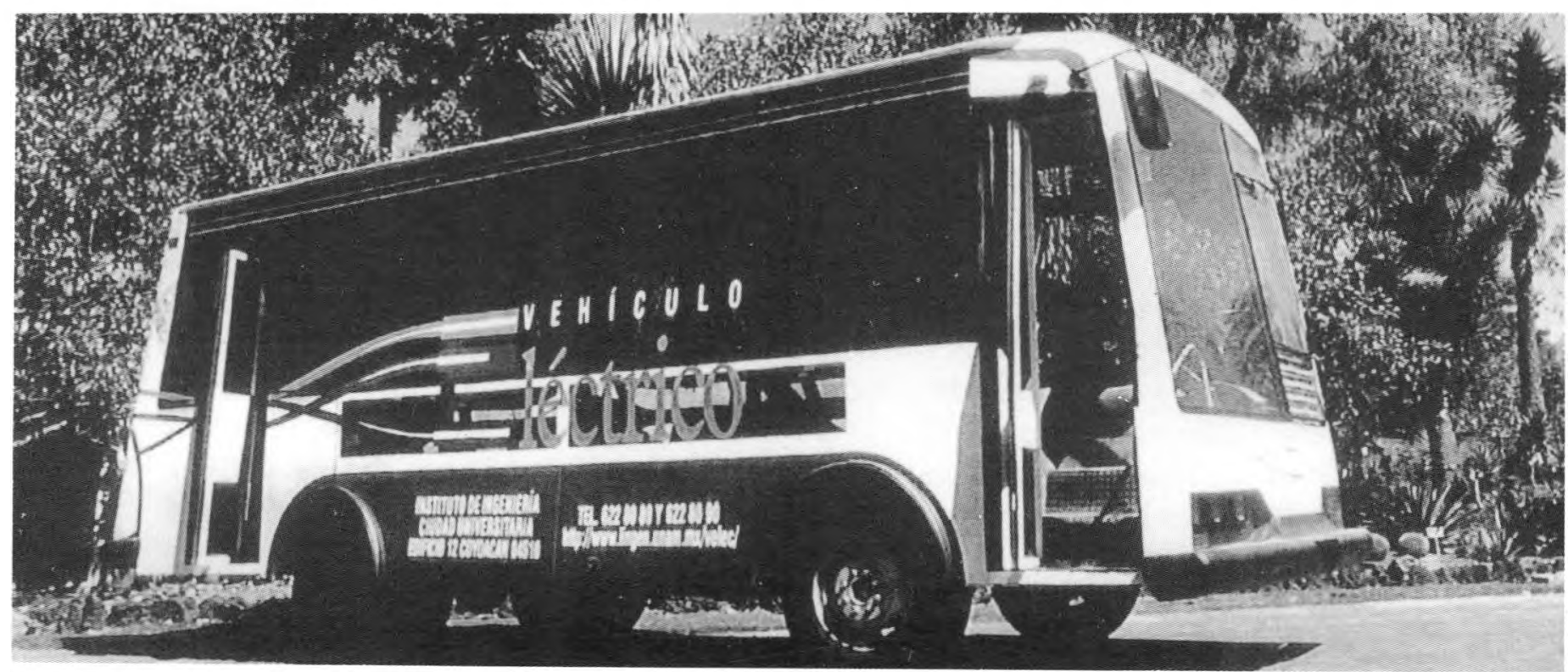

Figura 1. Vehículo Eléctrico UNAM (VE-UNAM) 
El VE-UNAM cuenta con un chasís ligero, estructurado con paneles de material compuesto tipo "sandwich", cuyas caras están hechas de varias capas de fibra de vidrio con diferentes orientaciones, embebidas en resina epóxica. Dichas caras están separadas por un núcleo de papel fenólico con geometría de panal de abeja e impregnado en resina.

El vehículo es impulsado por un motor de inducción, que se alimenta por corriente trifásica a $220 \mathrm{~V}$ y desarrolla una potencia continua de $22 \mathrm{KW}$, así como una potencia pico de $33 \mathrm{KW}$. Éste se ubica en la parte posterior del vehículo y va acoplado a un reductor de cadena con una relación de 3.62. La cadena empleada es Morse, tipo HV (High Velocity). La flecha de salida del reductor se acopla a una flecha cardán que transmite la potencia al diferencial del eje motriz, que es el trasero. La bomba hidráulica del sistema hidroneumático va acoplada directamente al motor de tracción, por lo que ambos giran siempre a la misma velocidad.

Para la tracción, se utilizan 52 baterías selladas de plomo ácido y otras 4 para los sistemas auxiliares, las cuales van colocadas sobre canastillas distribuidas a ambos lados del chasís. El peso total del vehículo con carga es de 7 toneladas y tiene una autonomía de $40 \mathrm{~km}$, una velocidad máxima de 40 $\mathrm{km} / \mathrm{hr}$ y una aceleración máxima de $0.6 \mathrm{~m} / \mathrm{s}^{2}$.

A continuación, se presentan las características principales del vehículo.

\section{Vehículo Eléctrico UNAM (VE-UNAM)}

\section{Motores y baterías}

Número de motores:

Potencia continua total:

Potencia total pico:

Número de baterías:

Tipo de baterías:

Otras características

Frenado: Regenerativo (hidroneumático)

Tracción: Trasera

Aceleración: $\quad 0.6 \mathrm{~m} / \mathrm{s}^{2}$ máximo

$\begin{aligned} \text { Velocidad máxima: } & 40 \mathrm{~km} / \mathrm{hr} \\ \text { Peso total con carga: } & 7,000 \mathrm{~kg} \\ \text { Capacidad: } & 30 \text { pasajeros } \\ \text { Autonomía: } & 40 \mathrm{~km}\end{aligned}$

\section{Electrobús}

El vehículo Electrobús (Figura 2), es otro minibús para 30 pasajeros que se diseñó en 1998 bajo un convenio con el Gobierno del Distrito Federal, que tuvo como finalidad el diseño de un prototipo de vehículo eléctrico autónomo que pudiera ser operado y evaluado por el Servicio de Transportes Eléctricos del Gobierno del DF.

El Electrobús tiene una carrocería estructurada con paneles de material compuesto tipo "sand-. wich" que forma un "monocasco", es decir, una estructura integral. Las ventajas que se derivan de esta construcción son de mayor seguridad al eliminarse elementos metálicos conductores de electricidad, resistencia a la corrosión, solidez resultante de la eliminación de juntas atornilladas o remachadas, así como el potencial de reducir peso.

El chasís que se utilizó es el de un vehículo Chrysler Ram 4000, el cual está diseñado para llevar el motor en la parte delantera con el volante de la dirección atrás del espacio ocupado por dicho motor lo cual no era aceptable para el caso del Electrobús. pues los motores de tracción se ubican debajo de la carrocería, entre los ejes. Ello requirió desplazar el volante y el reductor de sinfín y sector hacia el frente. e introducir una flecha para transmitir el par de salida de dicho reductor hasta el brazo Pitman.

El Electrobús tiene dos motores de tracción ubicados atrás del eje delantero. Transmiten su potencia a un reductor, semejante al del VE-UNAM, pero dual, de tal manera que se tienen dos catarinas de entrada, una por cada motor, enlazadas con catarinas en una flecha central de salida, la cual acopla a la flecha cardán que se extiende hacia atrás para conectar con el diferencial del eje motriz. Los motores son de corriente directa a $144 \mathrm{~V}$, y con ellos se dispone de una potencia continua de $44 \mathrm{KW}$ o $104 \mathrm{KW}$ pico. Las baterías son de plomo ácido para descarga profunda. Se tienen 54 para tracción y 6 para sistemas auxiliares. De igual forma que en el VE-UNAM, van distribuidas en . canastillas a ambos lados del chasís. 


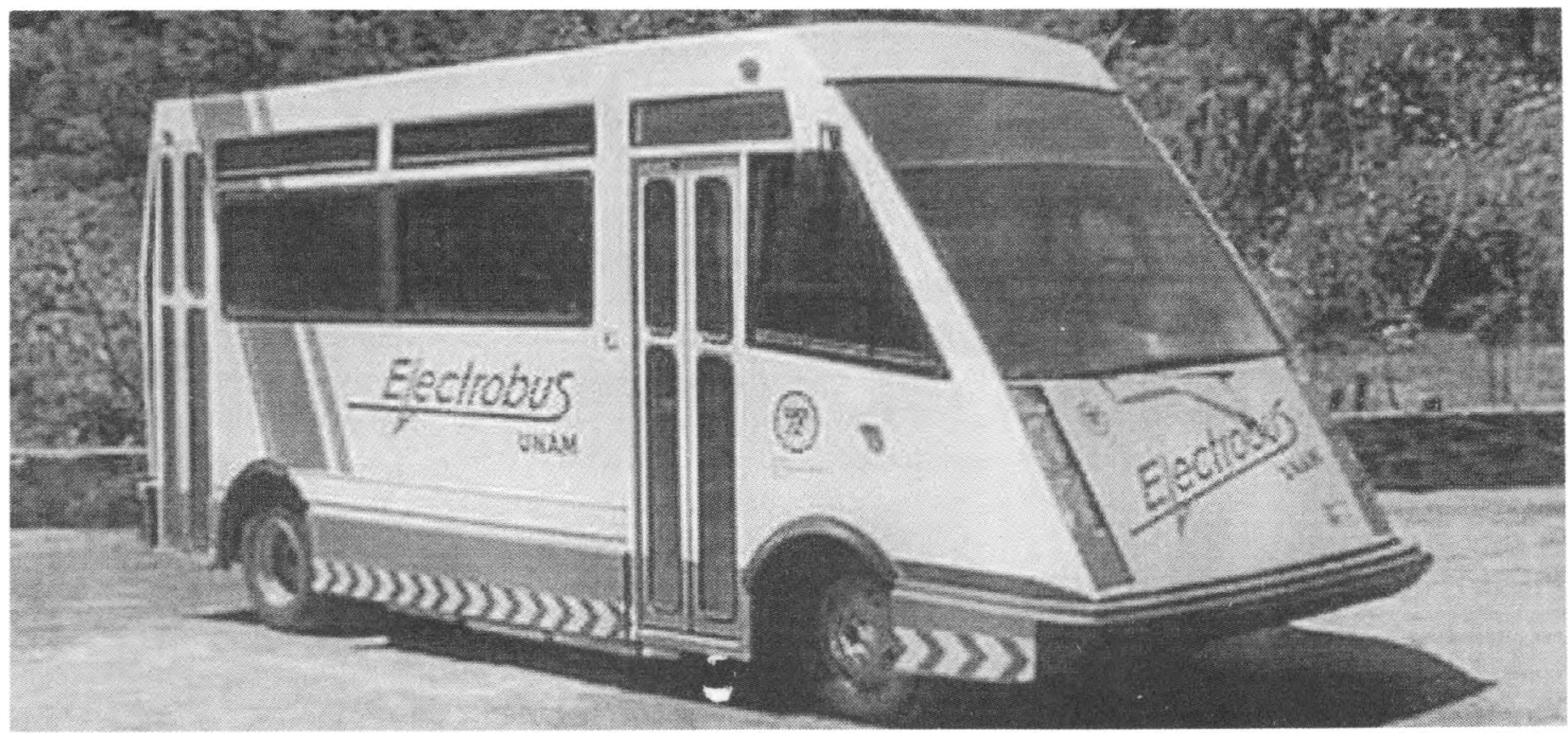

Figura 2. Electrobús

El peso total del vehículo con carga es de 7.5 toneladas. Tiene una autonomía de $60 \mathrm{~km}$, una velocidad máxima de $60 \mathrm{~km} / \mathrm{h}$ y es capaz de acelerar a $1.8 \mathrm{~m} / \mathrm{s}^{2}$ al arranque.

Ahora se muestran las características principales del vehículo.

\section{Vehículo Electrobus}

Motores y baterías

$\begin{aligned} \text { Número de motores: } & 2 \text { (corriente directa) } \\ \text { Potencia continua total: } & 44 \mathrm{~kW} @ 144 \mathrm{~V} \\ \text { Potencia total pico: } & 104 \mathrm{~kW} \\ \text { Número de baterías: } & 54 \text { para tracción } \\ & 6 \text { para servicios auxiliare } \\ \text { Tipo de baterías: } & \begin{array}{l}\text { Plomo-ácido, descarga } \\ \text { profunda de } 8 \text { V cada }\end{array} \\ & \\ \text { características } & \\ \text { Tracción: } & \text { Trasera } \\ \text { Aceleración: } & 1.8 \mathrm{~m} / \mathrm{s}^{2} \text { al arranque } \\ \text { Velocidad máxima: } & 60 \mathrm{~km} / \mathrm{hr} \\ \text { Peso total con carga: } & 7,500 \mathrm{~kg} \\ \text { Capacidad: } & 30 \mathrm{pasajeros} \\ \text { Autonomía: } & 60 \mathrm{~km}\end{aligned}$

\section{Vehículo repartidor Electrovira}

El vehículo repartidor Electrovira (Figuras 3 y 4) que actualmente se encuentra en proceso de fabricación, tiene la capacidad para hacer virar las ruedas delanteras en un intervalo de $180^{\circ}$. Para lograr este viraje, el mecanismo de la dirección incorpora dos amplificadores de engranes que actúan sobre sendos bastidores de viraje, (Chicurel E., 1999). También para este propósito y para mantener sencilla la transmisión, el vehículo tiene dos sistemas completos de propulsión, montados íntegramente en los bastidores de viraje (Figura 4). Cada unidad de tracción consta de un motor eléctrico, dos etapas de reducción de cadena y una rueda.

Gracias a este arreglo, no se requiere diferencial mecánico y su función, mantener los pares de tracción iguales en las dos ruedas motrices, se conseguirá mediante la inclusión de un controlador electrónico para cada motor. Ambos controladores recibirán una señal común de un potenciómetro incorporado al pedal acelerador.

Otros prototipos avanzados llevan los motores montados en los propios ejes de las ruedas, a diferencia de estos el Electrovira los lleva montados en los bastidores de viraje, que son parte de la masa suspendida, por tanto, quedan protegidos de los impactos causados por las irregularidades del terreno. 


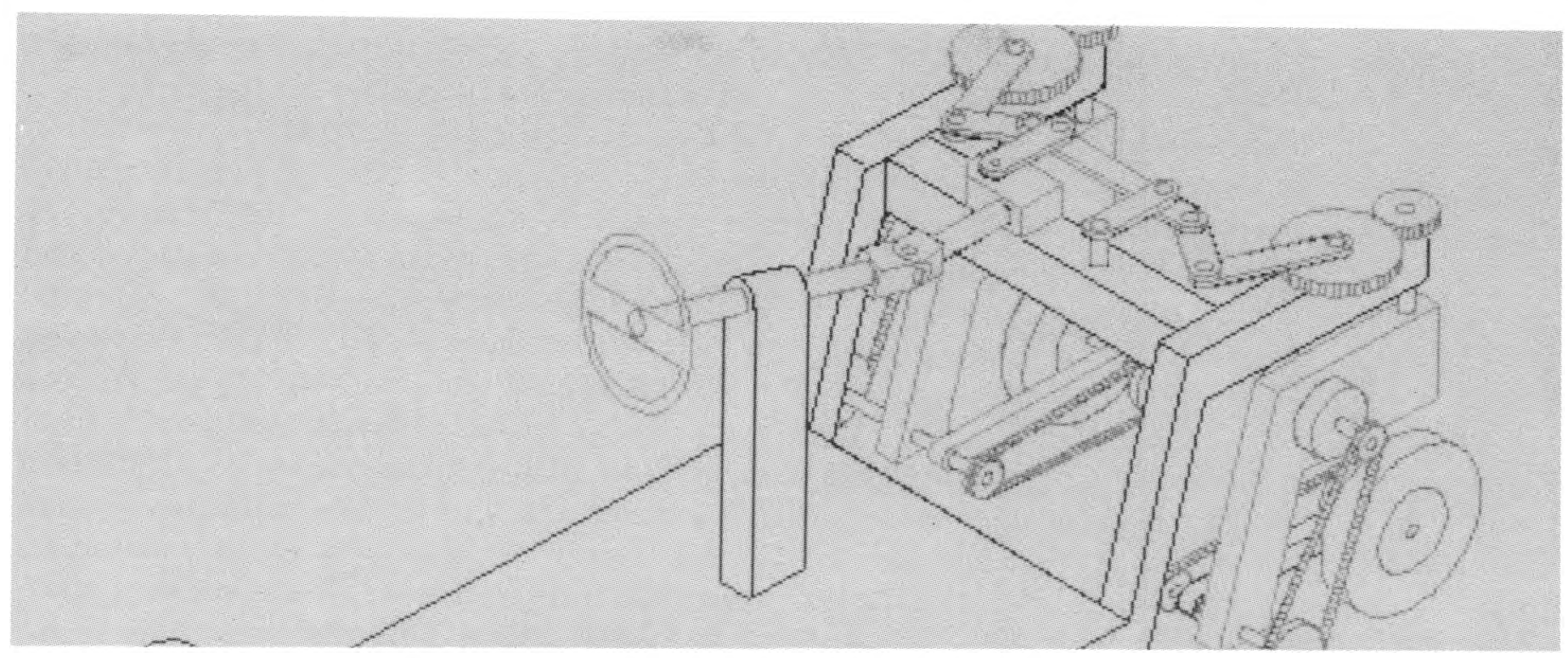

Figura 3. Concepto y arreglo general del vehículo Electrovira

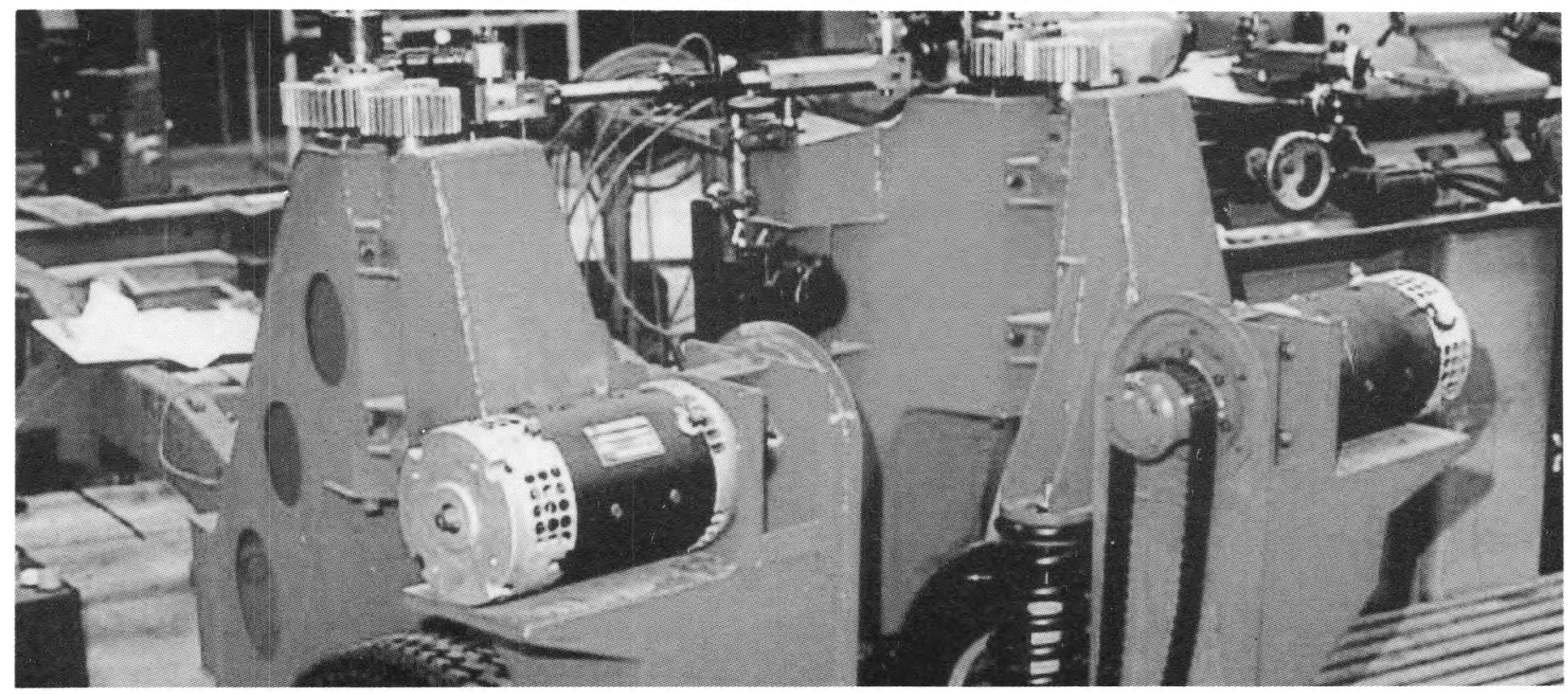

Figura 4. Vista frontal del Electrovira. Las dos unidades motrices aparecen en primer plano

\section{Vehículo Electrovira}

Motores y baterías

Número de motores: 2 (corriente directa)

Potencia continua total: 32 kW@120 V

Potencia total pico: $120 \mathrm{~kW}$.

Número de baterías: 20 para tracción

2 para servicios auxiliares

Tipo de baterías: Plomo-ácido, descarga profunda de $6 \mathrm{~V}$ cada una
Otras características

Tracción: Delantera

Intervalo de viraje de ruedas delanteras: $180^{\circ}$

Radio mínimo de viraje: 2.06 metros

Pendiente máxima: $14 \%$

Tiempo para acelerar de 0-75 km/hr: 22 segundos

Velocidad máxima: $\quad 75 \mathrm{~km} / \mathrm{hr}$

Peso total con carga: $\quad 3,000 \mathrm{~kg}$ 
DOI: http://dx.doi.org/10.22201/fi.25940732e.2002.03n3.011

Tanto en Japón como en Alemania, se han generado prototipos de vehículos e incluso automóviles comerciales con dirección en las cuatro ruedas, en términos generales, la idea es ganar estabilidad a alta velocidad y maniobrabilidad a baja velocidad, pero esto requiere de dos o tres modos de viraje, puesto que para alta velocidad las ruedas deben virar en fase y a baja velocidad en contrafase; y para ciertas maniobras conviene retener el viraje convencional. En un caso particular, los diseñadores de un camión de peso medio Nissan señalan que se gana considerablemente en maniobrabilidad, pero el "coleo" de la parte posterior del vehículo es un serio problema, Katoh et al. (1990). Sin embargo, a pesar de lo complicado que resulta el sistema con tres modos de viraje, en dicho vehículo no se alcanza la maniobrabilidad del Electrovira

\section{Vehículo Eléctrico de Reparto (VER)}

El vehículo eléctrico de reparto (VER) que se muestra en la figura 5, se desarrolló en las instalaciones del Centro de Diseño y Manufactura de la Facultad de Ingeniería y se realizó considerando un alto porcentaje de integración nacional en las partes que lo componen. Su diseño inició a mediados de 1998 con el apoyo de la Dirección General de Apoyo al Personal Académico de la UNAM (DGAPA), bajo el programa PAPIIT. Se contó también con la participación de alumnos del Centro de Investigaciones en Diseño Industrial de la Facultad de Arquitectura para el diseño de la carrocería, aspectos ergonómicos y funcionalidad del vehículo

El VER cuenta con dos motores de tracción acoplados a cada una de las llantas traseras, sustituyendo así, al diferencial mecánico convencional por uno eléctrico, a través del control de velocidad independiente de cada motor. Se hizo el diseño y construcción de un chasis en PTR al que se le aplicó un análisis y modelado por elemento finito. La carrocería se construyó en fibra de vidrio, haciendo primero el modelo a escala real en madera y luego moldes también en fibra de vidrio, de los cuales se hicieron las piezas definitivas. En el área de carga se utilizaron páneles de material compuesto para soportar los $1500 \mathrm{~kg}$ de carga útil.

Las baterías de plomo ácido para descarga profunda están localizadas a todo lo largo del vehículo y cuenta con espacio para 20 unidades de 6 volts cada una. Tiene un volumen útil de carga de poco más de $3 \mathrm{~m}^{3}$.

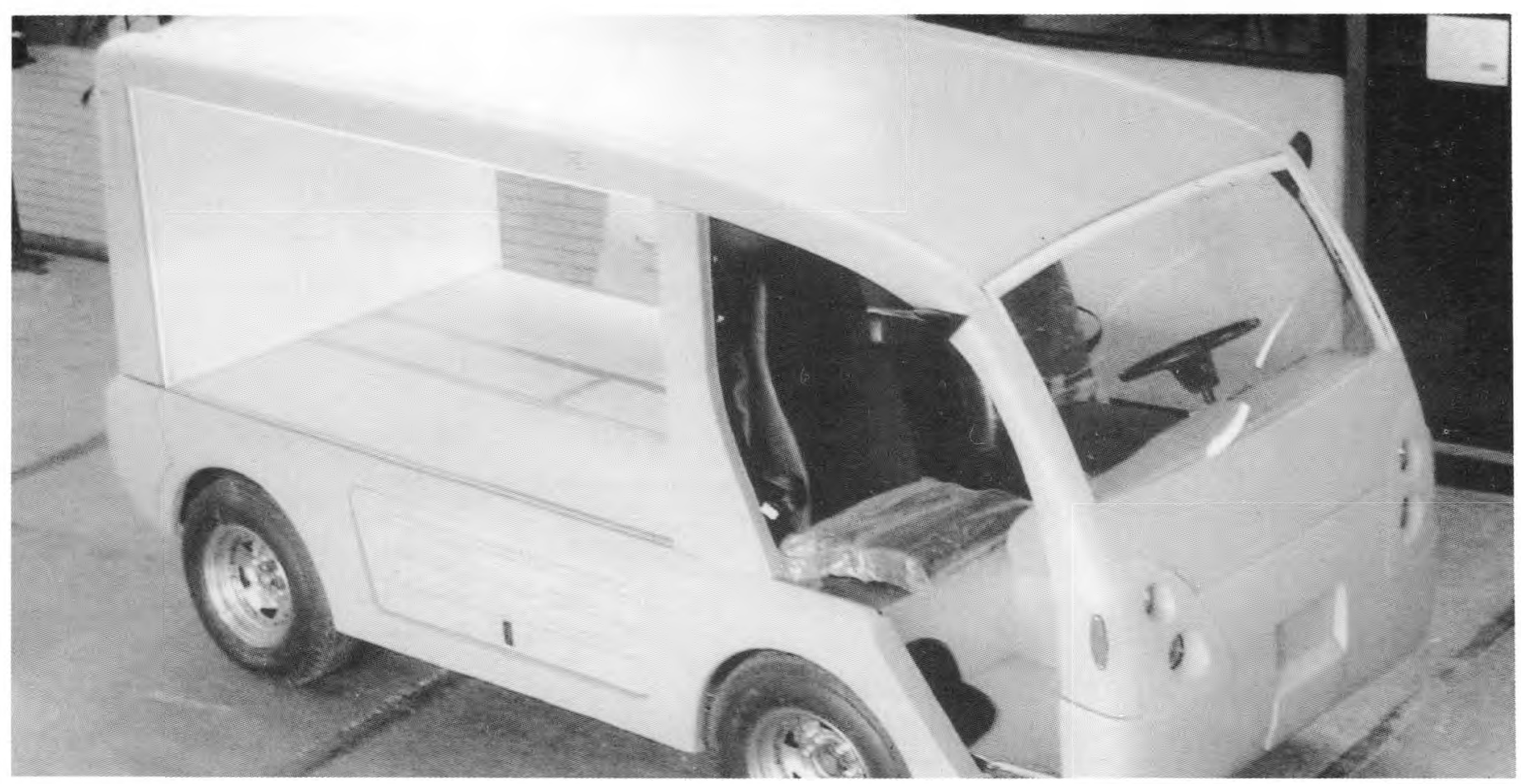

Figura 5. Vista del prototipo del Vehículo Eléctrico de Reparto 


\section{Vehículo Eléctrico de Reparto (VER)}

\section{Motores y baterías}

$\begin{aligned} \text { Número de motores: } & 2 \text { (corriente directa) } \\ \text { Potencia continua total: } & 28 \mathrm{~kW} @ 120 \mathrm{~V} \\ \text { Potencia total pico: } & 70 \mathrm{~kW} \\ \text { Número de baterías: } & 20 \text { para tracción } \\ & 1 \text { para servicios auxiliares } \\ & \begin{aligned} \text { Plomo-ácido, descarga } \\ \text { Tipo de baterías: }\end{aligned} \\ & \text { profunda de 6 V cada una }\end{aligned}$

\section{Otras características}

$\begin{aligned} \text { Tracción: } & \text { Trasera } \\ \text { Diferencial: } & \begin{array}{l}\text { Eléctrico con un motor en } \\ \text { cada rueda trasera con } \\ \text { tracción independiente }\end{array} \\ \text { Velocidad máxima: } & 60 \mathrm{~km} / \mathrm{hr} \\ \text { Autonomía: } & 60 \mathrm{~km} \\ \text { Capacidad de carga: } & 1500 \mathrm{~kg} \\ \text { Peso total con carga: } & 3,000 \mathrm{~kg}\end{aligned}$

\section{Otros proyectos en desarrollo}

Como consecuencia del diseño de los vehículos eléctricos en el Instituto de Ingeniería, se ha creado la necesidad de elaborar otros proyectos como:

1. Cargador inteligente para grandes paquetes de baterías.

2. Controlador electrónico dual.

3. Estudio del desempeño dinámico de baterías de plomo-ácido.

4. Sistema de monitoreo.

5. Búsqueda de nuevas alternativas de baterías.

El cargador inteligente para grandes paquetes de baterías, se desarrolló particularmente para el vehículo eléctrico UNAM y el Electrobús, ya que no fue posible encontrar un cargador con las características que requerían los bancos de baterías de estos vehículos. Este cargador está diseñado para suministrar hasta 40 Amperes de carga a un banco de baterías hasta de $200 \mathrm{Vdc}$ y requiere de una alimentación trifásica en 220 Vac. Actualmente está en operación con control manual y se trabaja en el desarrollo de la etapa de control automático con una computadora personal en la que se podrá programar el perfil de carga que el fabricante recomiende.

El controlador electrónico dual está en desarrollo y se diseña para controlar los dos motores de tracción del vehículo eléctrico de reparto. Este controlador deberá tener la capacidad de manejar en forma independiente a cada motor, a través de algoritmos de control que permitan la operación de los dos motores como diferencial eléctrico, en sustitución de un diferencial mecánico.

Para poder realizar el estudio del desempeño de los vehículos eléctricos y, en particular, el estudio dinámico de baterías de plomo-ácido, se realizó la conversión a tracción eléctrica de un vehículo. Volkswagen Sedán. Este estudio fue necesario, ya que para las baterías de este tipo, sometidas a una descarga dinámica como lo es un vehículo eléctrico, no ha sido sencillo obtener una metodología para conocer su estado de carga en todo momento, lo cual es de suma importancia para tener una idea de la distancia que se puede recorrer. En esta labor se tuvieron que diseñar sensores con amplificadores aislados ópticamente para poder introducir la información de manera segura en la computadora, a través de una tarjeta de conversión analógica-digital, también conocida como tarjeta de adquisición de datos. En la figura 6 se presentan los sistemas de acondicionadores de señal (amplificadores aislados) colocados en el. "Volts Wagen".

Otra actividad muy importante en la que el Instituto de Ingeniería está trabajando, es en la búsqueda de nuevas alternativas en baterías, ya que el mayor problema que tiene un vehículo eléctrico es su almacén de energía (baterías) que actualmente son muy pesadas y con poca energía, lo que limita el uso de estos vehículos a aplicaciones cuya necesidad de transporte no vaya más allá de 60 a 80 $\mathrm{km}$ de recorrido. Por lo anterior, se creó el Consorcio Mexicano de Baterías Avanzadas (COMBA) del cual, el Instituto de Ingeniería forma parte y es uno de los fundadores. 


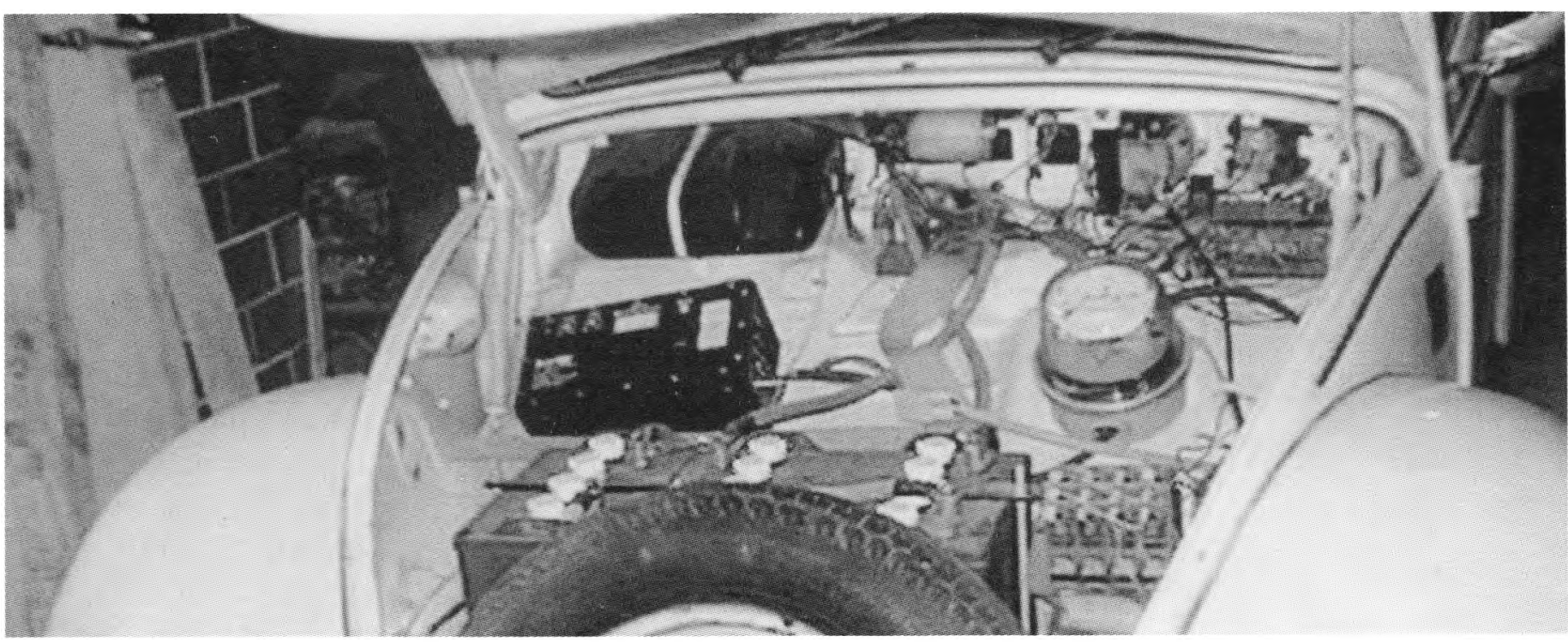

Figura 6. Volts Wagen con sistema de monitoreo instalado

Se ha puesto particular atención a la batería de Zinc-Aire, que es una de las que mejores características tiene. Ahora presentamos una tabla en la que se muestra la energía específica y costo de varios tipos de baterías, en la que se puede observar las bondades de las baterías Zinc-Aire.

\begin{tabular}{|c|c|c|c|}
\hline Tipo de batería & $\begin{array}{l}\text { energía } \\
\text { (Wh/kg) }\end{array}$ & específica & $\begin{array}{l}\text { costo } \\
\text { (\$/kWh) }\end{array}$ \\
\hline Níquel-Hidruro Metálico & 80 & & 550 \\
\hline Níquel-Cadmio & 60 & & 350 \\
\hline Litio-Ion & 90 & & $1,000+$ \\
\hline Plomo-Ácido & 44 & & 150 \\
\hline Zinc-Aire & 180 & & 150 \\
\hline
\end{tabular}

Fuente: "Report of California Air Resources Board Battery Technical Advisory Panel

\section{Conclusiones}

El programa de desarrollo de vehículos eléctricos. autónomos, creado en el Instituto de Ingeniería de la UNAM hace ocho años, ha rendido frutos concretos como los prototipos de dos minibuses con características diferentes, dos vehículos de reparto, uno de ellos con gran maniobrabilidad, y otros desarrollos como el cargador de grandes paquetes de baterías, así como el sistema de monitoreo.

Por su parte, el Vehículo Eléctrico UNAM ha operado en diversos recorridos en Ciudad
Universitaria y por las calles de la Ciudad de México. El sistema de frenado hidroneumático se ha probado en forma preliminar en el laboratorio y se esperan iniciar en breve las pruebas para su evaluación en campo.

El Electrobús ha acumulado varios cientos de kilómetros en recorridos de prueba, incluyendo una etapa de varios días transportando pasajeros en una ruta que comprende partes de la primera y segunda secciones del Bosque de Chapultepec. En breve, iniciará una etapa de prueba de seis meses ofreciendo servicio de transporte de pasajeros en el circuito de Ciudad Universitaria.

Por otra parte, el vehículo Electrovira se encuentra en una etapa avanzada de fabricación. ya se montaron en el chasís el mecanismo de la dirección, los bastidores de viraje y las unidades de tracción. El mecanismo de la dirección está proporcionado de acuerdo a una síntesis por optimación llevada a cabo por computadora y una apreciación cualitativa, al operar el volante, indica que se cumple con la ley de viraje, es decir, que en una curva las cuatro ruedas describen trayectorias de arcos de círculos concéntricos.

El Vehículo Eléctrico de Reparto está prácticamente terminado para iniciar etapas de pruebas y ajustes. Se han realizado varias pláticas con fabricantes del ramo, quienes mostraron interés por el vehículo y ofrecieron posibilidades de tener acuerdos para su explotación comercial. 
La participación del Instituto de Ingeniería en el COMBA ha rendido frutos concretos, como la prueba de una celda de combustible de Zinc-Aire y actualmente está en proceso un proyecto para la instalación y prueba de baterías de Zinc-Aire en un vehículo eléctrico de reparto de mercancías.

\section{Agradecimientos}

Los autores hacen patente su reconocimiento al Consejo Nacional de Ciencia y Tecnología por el apoyo recibido para la realización de varios aspectos del desarrollo de los vehículos, asimismo, al Servicio de Transportes Eléctricos del Distrito Federal por su participación y financiamiento en el proyecto Electrobús y a la Dirección General de Apoyo al Personal Académico de la UNAM, la cual patrocinó actividades precursoras del trabajo que aquí se presenta.

\section{Referencias}

Chicurel E. (1999). A $180^{\circ}$ Steering Interval Mechanism. Mechanism and Machine Theory, Vol. 34, No. 3, 421-436.
Chicurel R. y Lara P. (1996). Control of a Hybrid Propulsion System. Instrumentation and Development, Vol. 3, No. 6, 3-7.

Chicurel R. (1999). A Compromise Solution for Energy Recovery in Vehicle Braking. Energy, Vol. 24, No.10, 29-34.

Chicurel R., Carmona G. y González, A. (2001). Sistema hidroneumático para recuperación de energía de frenado. Memoria del VII Congreso Sociedad Mexicana de Ingeniería Mecánica Celaya, Guanajuato, 37-41.

Hammerström L. (1984). VTS-Hydrostatic Transmis- . sion. Experiences From the First Field Test. Proc. International Symposium Advanced and Hybrid Vehicles. University of Strathclyde, 235-41.

Katoh H., Torizuka T., Minami K., Hata N. y Watanabe Y. (1990). Development of a Medium Duty Truck with a Mechanical Linkage Type Four Wheel Steering System. SAE Transactions, Vol. 99, No. 2, 870-876.

Martini S. (1984). The M.A.N. Hydrobus. A Drive Concept with Hydrostatic Brake Energy Recovery. Proc. International Symposium Advanced and Hybrid Vehicles. University of Strathclyde, 227-34.

\section{Semblanza de los autores}

Germán Carmona-Paredes. Es ingeniero mecánico electricista egresado de la Facultad de Ingeniería de la UNAM en 1993. Trabaja en el Instituto de Ingeniería de la UNAM en proyectos sobre el diseño de vehículos eléctricos. particularmente en los sistemas de control de motores eléctricos y nuevas tecnologías de baterías.

Ricardo Chicurel-Uziel. Obtuvo el título como ingeniero mecánico de Cornell University en 1953, el de maestro en ingeniería en 1957 y el doctorado de Princeton University en 1959. Fue profesor de mecánica teórica y aplicada en Virginia Polytechnic Institute and State University de 1959 a 1970. Ha sido investigador del Instituto de Ingeniería de la UNAM desde 1970. Fue presidente de la Academia Nacional de Ingeniería de 1983 a 1985.

Enrique Chicurel-Uziel. Es ingeniero mecánico por la Universidad de Cornell, obtuvo la maestría en la Universidad Washington y el doctorado en la Universidad de Wisconsin. Es miembro de Sigma Xi y miembro fundador de la ANI. Trabajó en IEM y la Compañía de Luz y Fuerza de la Ciudad de México. Fue profesor en la Universidad de Boston y en la Universidad de las Américas. En la UNAM, conjuntamente con el Ing. Alberto Camacho Sánchez, inició tanto el posgrado como las investigaciones en ingeniería mecánica.

Filiberto Gutiérrez-Martínez. Ingeniero industrial mecánico egresado del Tecnológico de la Laguna en 1975. Obtuvo una maestría en ingeniería térmica en el ITESM, Campus Monterrey en 1977. Es investigador del Instituto de Ingeniería, UNAM, donde labora desde 1978. Ha impartido cursos a nivel licenciatura y posgrado en la Facultad de Ingeniería, así como en el posgrado de la Facultad de Arquitectura, UNAM. Su campo de actividades incluye el trabajo experimental relacionado con diversas aplicaciones fototérmicas de la energía solar y con el desarrollo de vehículos de alta maniobrabilidad. 
